\title{
Interlaced dynamics of density waves and vortices in self- gravitating discs
}

\author{
G. R. Mamatsashvili ${ }^{1, a}$ \\ ${ }^{1}$ Faculty of Exact and Natural Sciences, Tbilisi State University, II. Chavchavadze ave. 3, Tbilisi 0179, \\ Georgia
}

\begin{abstract}
Latest developments in the dynamics of density waves and vortices in selfgravitating protoplanetary discs is reviewed. It is well established by now that in discs, vortices are dynamically coupled with density waves due to the disc's differential rotation, or shear. On the other hand, density waves play a central role in the theory of self-gravitating discs and recently revealed their coupling with vortices implies that the latter can also be subject to self-gravity effects, thus taking active part in defining overall dynamics of self-gravitating discs. We describe the specific features of vortex dynamics and evolution in self-gravitating discs with and without driving by baroclinic or Rossby wave instabilities and point out differences between these two case.
\end{abstract}

\section{Introduction}

Research in the field of dynamics and evolution of protoplanetary discs is mainly focused on studying two basic kinds of perturbations - density waves and vortices - that occur in them. At an earlier stage of evolution, when the disc is massive enough for its self-gravity to be important, large scale spiral density waves commonly emerge as a result of gravitational instabilities within the disc. It has been known for some time that these waves play an important twofold role. Firstly, they act to transport angular momentum outwards, allowing disc mass to accrete onto the protostar, and most likely are a primary transport mechanism during the earliest stages of star/disc formation [e.g., see reviews 1, 2]. Secondly, density wave crests may well represent sites of planet formation [3, 4].

Typically, effective radiative (realistic) cooling times in self-gravitating discs are too long, much larger than Gammie's fragmentation threshold value $\sim 3 \Omega^{-1}[5]$ ( $\Omega$ is the angular velocity of disc rotation), to permit break-up of the disc (at radii < 100AU) by its own self-gravity [6, 7]. Consequently, balance is established between heating due to gravitational instability and cooling (self-regulation mechanism, $[8,9])$. Discs are expected to stay in this self-regulated, quasi-steady, so-called gravitoturbulent state for hundreds of orbits. In this case, Toomre's stability parameter hovers on the margin of gravitational instability. The properties of this state were numerically investigated in a large number of papers [e.g., 5, 6, 9-15, and references therein]. A general dynamical picture, as mentioned above, is that a spiral structure, sustained by the self-regulation mechanism, develops in the disc and

\footnotetext{
a e-mail: george.mamatsashvili@tsu.ge
} 


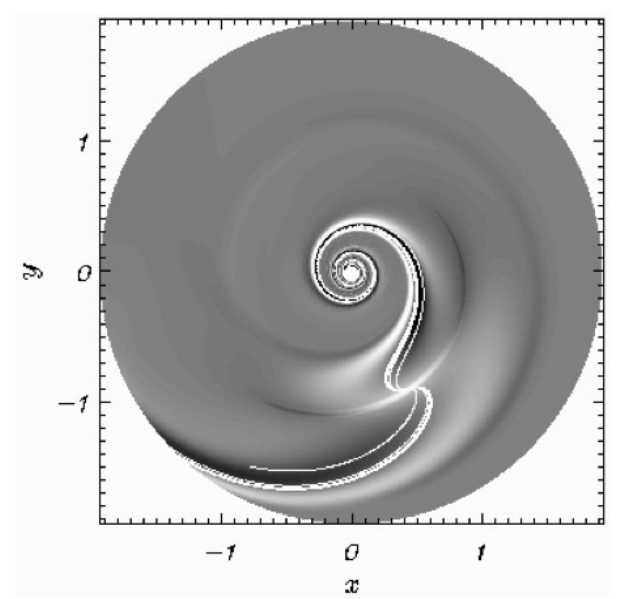

Figure 1. Emission of spiral density waves by a localized vortex embedded in a non-self-gravitating Keplerian disc. Adapted from [16].

transports angular momentum outwards through gravitational and hydrodynamic stresses, thereby allowing matter to accrete onto the central star. All the angular momentum transport in this case has been attributed solely to spiral density waves.

Another perturbation type in discs - vortical mode - has received much attention in recent years, partly because of its connection with planetesimal formation. Latest studies demonstrated that vortices can arise in both self-gravitating and non-self-gravitating discs provided there is some localized radial structure in the disc triggering Rossby wave instability (RWI) whose non-linear development leads to sustained vortex formation [17-20]. Alternatively, vortices can also be generated as a result of other hydrodynamic instabilities, such as baroclinic instability [21, 22] or Kelvin-Helmholtz instability of seed small scale vorticity [23]. Although the dynamics and evolution of vortices in nonself-gravitating discs has been under active investigation for a while, since the pioneering idea of their role in planet formation process by [24], systematic studies of vortex dynamics under the influence of disc self-gravity appeared only few years ago [25-28]. It had been usually thought before that spiral density waves are the only perturbation type that can be present in self-gravitating discs and the possibility of existence of other modes - vortices - also participating in the overall disc dynamics had been left out of consideration. In other words, almost all studies on self-gravitating disc dynamics concentrated primarily on the dynamical activity of spiral density waves. Since vortices play an important role in non-self-gravitating discs, it was natural to look for them and analyze their nonlinear dynamics in self-gravitating discs too. In non-self-gravitating discs, it was shown that vortices can be (linearly) coupled with and excite spiral density waves due to the disc's differential rotation [16, 29, 30, see Fig. 1]. This coupling between spiral density waves and the vortical mode is even more efficient/stronger in the presence of self-gravity [31]. Moreover, a linear analysis carried out in the latter study demonstrated that in fact vortical mode can also be subject to the effect of self-gravity and hence exhibit gravitational instability with amplification factors comparable to those of density waves. This clearly implies that vortical mode is equally important together with density waves in forming a complete dynamical picture of self-gravitating discs.

Subsequent non-linear dynamics and evolution of vortices vary dramatically between non-selfgravitating and self-gravitating cases. In non-self-gravitating discs, random small scale anticyclonic (i.e., rotating opposite to disc's Keplerian rotation) vortices, smoothly merge into each other and increase in size, evolving in this way into coherent large-scale structures [e.g., 18, 21, 22, 32-36]. When the vortex sizes reach a few scale height, compressibility becomes important, causing them 

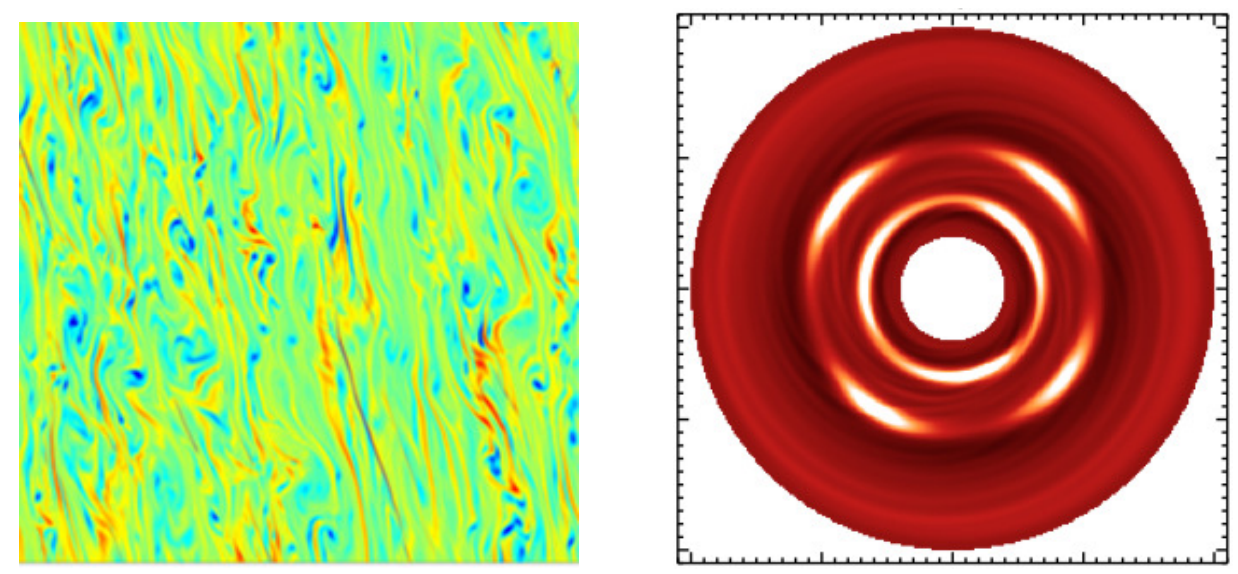

Figure 2. Vortices in a self-gravitating disc represented by two setups: the local shearing sheet model without radial structure residing in a self-regulated gravitoturbulent state (left) and a global model supporting the RWI near the dead zone edges, but remaining laminar despite self-gravity (right). In gravitoturbulence, the vortices are smaller scale, irregular, short-lived structures, recurring after each couple of disc orbital times. On the other hand, the vortices driven by the RWI in the global model, remain long-lived and regular, but are reduced in size due to the effect of self-gravity compared with their non-self-gravitating counterparts. Adapted from [25] and [26].

to emit shocks of spiral density waves due to the above-mentioned shear-induced mode coupling phenomenon [35-37]. This leads to a slow decay of vortices on the timescale of several hundred orbital periods. In spite of shock dissipation, anticyclonic vortices can be considered long-lived, coherent structures in discs. In non-self-gravitating discs, particle trapping capability of such vortices was also investigated [e.g., 26, 38, 39]. It has been shown that a smooth, sufficiently long-lived vortex is indeed able to effectively trap dust particles in its core, possibly accelerating planetesimal formation.

The above dynamical picture changes if the disc is self-gravitating. Vortex merging is resisted by the action of self-gravity and smaller size vortices are favoured more [25-28]. In some sense, selfgravity acts to oppose inverse cascade of power to larger scales (i.e., to smaller wavenumbers in the spectral space), as it happens in the non-self-gravitating case, and to scatter it to smaller ones. The local shearing sheet simulations of radially unstructured (i.e., without driving by the baroclinic or RW instabilities) discs indicate that vortices in a quasi-steady gravitoturbulence are transient short-lived structures undergoing repeated cycles of formation, growth to sizes comparable to the local Jeans length, and eventual shearing and destruction by self-gravity and Keplerian shear [25]. This process lasts a few orbital periods, and results in a very different to the non-self-gravitating case evolutionary picture with many small, less organized irregularly-shaped vortices at various stages of evolution (Fig. 2), rather than the relatively larger scale, well organized vortices gradually growing via slow mergers. On the other hand, in global simulations [26-28], discs are radially structured and as a result develop relatively coherent, regular and longer-lived vortices due to the RWI (vortensity minimum), though remain laminar, without gravitoturbulence, despite being self-gravitating (Fig. 2). Subject to self-gravity effect, these vortices have smaller azimuthal extent (higher azimuthal wavenumbers) in comparison with those occurring in non-self-gravitating discs. It is noteworthy that vortices in both local and global simulations share a common feature - they produce overdensities at their centres coinciding with the minima of Toomre's parameter $Q$. These overdensities are overlaid on the 
density variations due to shocks of density waves emitted by these vortices. The gaseous overdensities/overpressures are thought to aid planet building process. Because the gravitational instability cannot form giant planets directly via fragmentation [7, 15], indirect means of planet formation accumulation of dust particles in gaseous density structures associated with vortices and coupled to them density waves (shocks) - could be at work in self-gravitating discs.

Trapping of dust particles by spiral density waves and the possibility of planetesimal formation via this process in self-gravitating discs were explored in both global [3] and local shearing sheet [4] simulations. It was demonstrated that density waves are efficient at trapping solids, especially those with stopping times comparable to the local orbital period. This can eliminate two problems currently challenging theories of planet formation. Firstly, concentration of solid material trapped within a spiral arm can become locally much larger than the average within the disc, so that particle agglomerations there will collapse due to own self-gravity, possibly leading to faster creation of planetesimals. Secondly, particle trapping in local pressure maxima can also prevent growing objects from rapidly drifting inwards and being lost from the disc.

Dust trapping by pressure maxima associated with (anticyclonic) vortices is based on a similar physical mechanism and, as mentioned above, has been extensively covered, though only in the context of non-self-gravitating discs. So, the role of vortices in trapping dust in self-gravitating discs is lesser understood and needs further investigation. Particle concentration by vortices in the presence of disc self-gravity has been first addressed by [26] through global disc simulations. However, in their setup vortices were generated by the RWI in an otherwise laminar disc and had long-lived regular nature, as in the non-self-gravitating case, though smaller azimuthal size due to the effect of selfgravity. In this respect, it would be interesting to explore how effective transient vortical structures in a gravitoturbulent state are in collecting dust particles.

In the remainder of this paper, we present latest results on the effect of self-gravity on the evolution of vortices. Namely, we compare the evolution of a single vortex in non-self-gravitating and self-gravitating cases in the shearing sheet approximation using numerical simulations. We demonstrate that without self-gravity, an initial vortex undergoes non-linear adjustment, radiating density waves and reducing in size. By contrast, in the presence of self-gravity, the vortex gets destroyed by gravitational instability, because of producing density enhancement, and a gravitoturbulent state ensues with multiple much smaller, elongated, diffuse vortical structures. This is in agreement with previous above-mentioned results that self-gravity favours smaller (of the order of Jeans length) vortices in discs.

\section{Dynamics of a localized vortex with self-gravity}

To analyze the evolution of a localized vortex, following other analogous studies [5, 25, 40], we adopt the 2D local shearing sheet model with self-gravity, constant cooling time prescription and ideal gas equation of state (with $\gamma=2$ ), so the dynamical equations solved here are essentially the same as those given in these papers and hence are omitted. The constant cooling time $\tau_{c}=20 \Omega^{-1}$, so that the disc does not fragment and enters a saturated gravitoturbulent state. The cooling is an essential factor in self-gravitating disc simulations, without it dissipation in strong shocks resulting from the gravitational instability would quickly heat up the disc and thus switch off self-gravity effect.

A central quantity in $2 \mathrm{D}$ configuration is the vertical component of potential vorticity (PV):

$$
I=\frac{1}{\Sigma}\left(\frac{\partial u_{y}}{\partial x}-\frac{\partial u_{x}}{\partial y}+(2-q) \Omega\right)
$$

where $u_{x}$ and $u_{y}$ are the components of the velocity relative to the background Keplerian shear flow, $\Sigma$ is the surface density and $\Omega$ is the disc's angular velocity. The shear parameter $q=1.5$ for the Kep- 


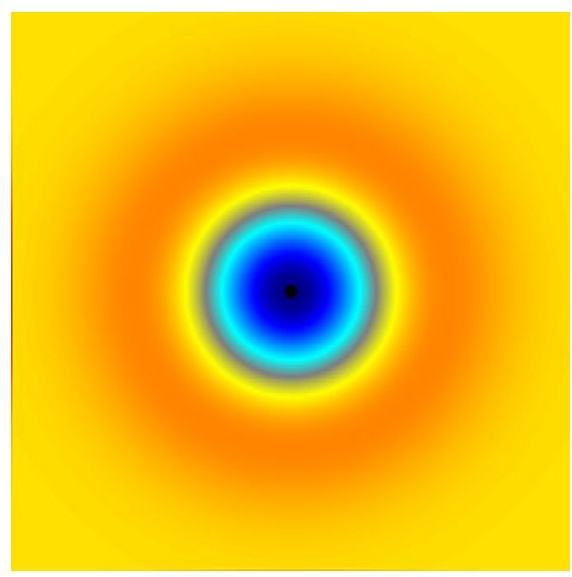

Figure 3. Distribution of PV with coordinates corresponding to the initial circular vortex. It is anticyclonic with negative PV $\left(I<0\right.$, blue area), which has a minimum, $I_{\min }=-0.7$, at the centre.

lerian rotation. The PV is often used for characterizing the formation of coherent structures (vortices) in disc shear flows [35].

We ran non-self-gravitating and self-gravitating setups starting out with the same initial condition - a localized anticyclonic vortex imposed on top of the background Keplerian shear flow at the centre of the computational domain (Fig. 3),

$$
u_{x}=a y e^{-\frac{x^{2}+y^{2}}{l^{2}}}, \quad u_{y}=-a x e^{-\frac{x^{2}+y^{2}}{l^{2}}},
$$

where the parameters $a$ and $l$ characterize, respectively, the vortex amplitude, that is, the PV at the vortex eye (which is negative for an anticyclonic vortex) and vortex size. We take $l=8.0$ and $a=0.6$, which corresponds to a minimum PV at the vortex centre, $I_{\min }=-0.7$ so that the vortex is in the non-linear regime from the outset (these quantities are normalized as in [25]). The surface density and internal energy are not perturbed initially. For the run with self-gravity, the initial Toomre's parameter is $Q_{0}=c_{s 0} \Omega_{0} / \pi G \Sigma_{0}=1.0$, where $c_{s 0}$ is the initial equilibrium sound speed in the disc. The computational domain in the $(x, y)$ plane is a square $-L / 2 \leq x, y \leq L / 2$, divided into $N \times N$ grid cells; we take $L=20$ and $N=512$. To follow the subsequent time-development of this initial vortex configuration with and without self-gravity, we numerically integrate the governing equations within this domain using a version of the ZEUS code adapted for the shearing sheet geometry [5, 35].

\subsection{Non-linear evolution}

Figure 4 shows the outcomes of the non-self-gravitating and self-gravitating runs for the same initial vortex configuration described above. First consider the non-self-gravitating case. The vortex is first sheared by the disc's differential rotation, but eventually settles down (adjusts) into a stable configuration with reduced size that survives for many orbital periods (Fig. 4, left panel). It owes survival to anticyclonic nature; cyclonic vortices rotating with the disc, opposite to the Keplerian shear, would get quickly sheared out [e.g., 33, 35, 37]. During the adjustment process, spiral-density waves, propagating in the opposite directions, are emitted from the vortex because of the above discussed sheared-induced vortex-wave coupling phenomenon. Afterwards, non-linearity causes these waves to turn into shocks, whereas the vortex itself, which has undergone the adjustment, corresponds to the central overdense region. The dynamical picture of vortex evolution (adjustment) in non-selfgravitating discs, presented here in the local shearing sheet model, is in good agreement with that reported in similar global simulations in [37]. 

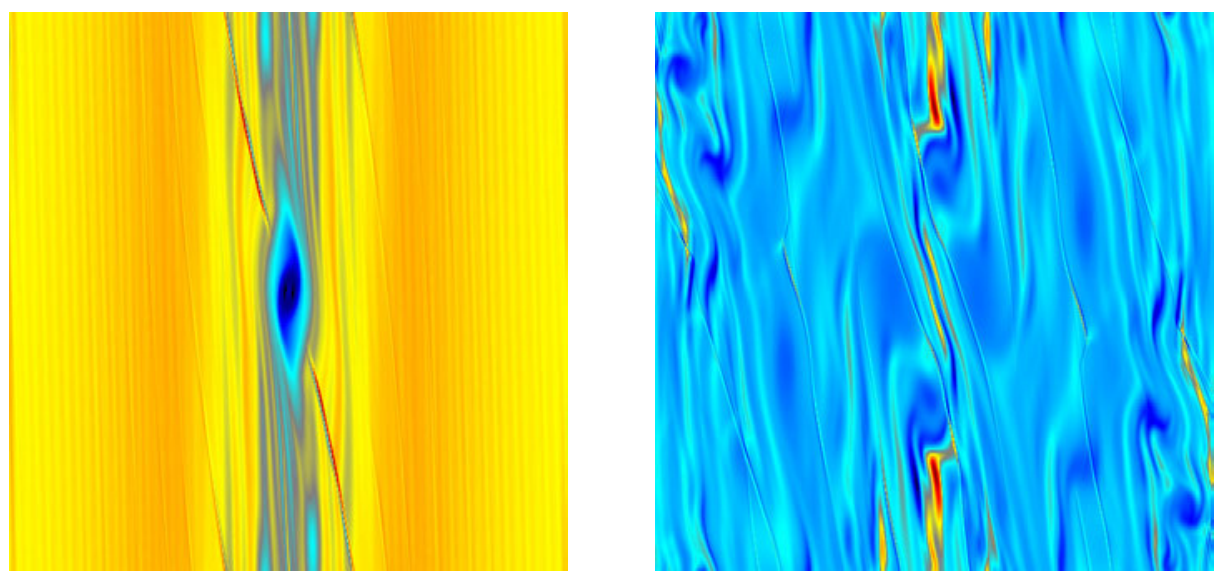

Figure 4. Subsequent evolution of a single vortex from Fig. 3 in non-self-gravitating (left) and self-gravitating (right) discs in the shearing sheet model. Shown here is the PV field with blue corresponding to anticyclonic (negative PV, $I<0$ ) and yellow to cyclonic (positive PV, $I>0$ ) regions. In non-self-gravitating discs, the initial vortex undergoes adjustment, reducing in size and emitting density waves, but stays localized and survives. By contrast, in the self-gravitating case, the original vortex gets destroyed by the effects of shear and self-gravity after a few orbits. The outcome in this case is a gravitoturbulence with multiple small-scale transient vortices.

In the presence of self-gravity, the disc cools down at the beginning, the $Q$ parameter decreases and individual spatial fourier harmonics (shearing waves) contained in the vortex undergo sizable swing amplification due to the increased effect of self-gravity. The transient growth of these vortical shearing waves is inevitably accompanied by the generation of density waves. [29, 31]. Enhancement in the surface density around the vortex eye induced by the growth of separate harmonics eventually becomes gravitationally unstable, causing the destruction of the initially imposed vortex. As a consequence, in the PV field, the nicely shaped initial localized 'blob' of anticyclonic vorticity at the domain centre is replaced by azimuthally elongated, diffuse and irregular regions of positive (cyclonic) and negative (anticyclonic) PV filling the whole domain (Fig. 4, right panel). Density waves generated by the vortex turn into strong shocks that propagate along the domain and heat up the disc. After a couple of orbital times, this shock heating comes into balance with cooling and, as a result, the disc settles down in a self-regulated, quasi-steady gravitoturbulent state, as typical of self-gravitating disc simulations with imposed cooling $[5,9,13]$. In this state, all the averaged values of characteristic quantities, energy, sound speed, transport parameter $\alpha$ are nearly constant with time. Subsequent dynamics and evolution of PV in the gravitoturbulence follows a pattern described in [25]. Namely, in this state, vortices appear as transient structures undergoing recurring phases of formation, growth to sizes comparable to a local Jeans scale and eventual shearing and destruction due to the combined effects of self-gravity and shear. Each such cycle typically lasts about a few orbital periods. As a result, in self-gravitating discs residing in a gravitoturbulent state, the overall dynamical picture of vortex evolution is irregular, consisting of many transient vortices at different evolutionary stages and, therefore, with various sizes up to the local Jeans scale.

Thus, the dynamics of a localized vortex in self-gravitating discs differ dramatically from that in non-self-gravitating ones, which is more often studied in the literature. In the non-self-gravitating case, vortex undergoes non-linear adjustment, but stays localized and is stable. By contrast, selfgravity generally prevents vortex development and disrupts, or breaks it up into smaller scale tran- 
sient irregularly shaped vorticies within dynamical/orbital time. Note that we have considered here a spatially uniform equilibrium without any baroclinic source of PV. In some global 2D simulations of self-gravitating discs characterized by a certain radial structure, as discussed in the Introduction, the emergence and long-term survival of vortices were observed primarily due to continual driving by the RWI [26, 28]. However, the disc setups considered in these studies somewhat differ from what is typically adopted in most simulations of self-gravitating protoplanetary discs [e.g., 5, 6, 9, 11, 13], in particular, there was no cooling function, which is largely responsible (in combination with selfgravity) for the onset of self-regulated grvaitoturbulence, and hence these discs tend to remain laminar despite being self-gravitating.

\section{Summary and conclusions}

In this proceeding article, we briefly reviewed specific dynamics and evolution of vortices in selfgravitating discs and brought out key features that make it different from more often studied vortices in non-self-gravitating discs. First of all we should note that in both cases, vortices are coupled with and generate density waves due to the linear mode coupling phenomenon induced by disc flow shear. This coupling is enhanced by disc self-gravity, implying that vortices can be dynamically as important as density waves in forming a complete picture of self-gravitating disc's overall dynamics. The main result of the non-linear development of vortices under disc self-gravity, be it a single localized vortex or more realistic random distribution of potential vorticity, is that, vortices become smaller scale structures under the influence of self-gravity compared to those in non-self-gravitating discs. They can no longer grow via merging into larger vortices (inverse cascade), or in other words, vortex merging is resisted, because self-gravity opposes an inverse cascade of power in wavenumber space and in this way more favours smaller scale vortices. In self-gravitating discs without baroclinic driving, vortices tend to be irregular transient structures recurrently forming and disappearing on orbital timescale, whereas with baroclinic driving (e.g., the RWI) they can live much longer and are more regular and well-shaped structures. This latter result has been obtained from global simulations of self-gravitating discs where the RWI is operating, but disc cooling is absent. In this respect, it would be interesting to investigate whether the RWI is still capable of competing with the destructive effect of self-gravity and producing long-lived regular vortices in the presence of cooling that leads to gravitoturbulence.

Another noteworthy property of anticyclonic vortices with self-gravity is that they produce much stronger overdense/overpressure regions on top of the shock waves than non-self-gravitating ones. This opens the door to the possibility of planetesimal formation in the earlier stages of protoplanetary discs when their self-gravity plays a role. Particle concentration only by density waves originating from gravitational instability was studied in $[3,4]$. As a sequel to this, it would be interesting to analyze particle trapping by overdensities due to vortices that are superimposed on density variations due to larger scale shocks of density waves in a self-regulated gravitoturbulent state. However, generally short-lived and transient nature of vortices in this case may somewhat hinder an efficient capture of dust particles. The goal would be to compare levels of particle concentrations trapped in transient vortices and in density waves. This will help us to understand how planet formation may proceed in non-fragmenting self-gravitating discs.

\section{Acknowledgments}

I would like to thank the organizing committee of the meeting "Instabilities and Structures in Protoplanetary Discs" held in Marseille, France, on 17-20 September, 2012, for inviting me and the Rustaveli National Science Foundation, Georgia, for financial support. 
EPJ Web of Conferences

\section{References}

[1] F.C. Adams, D.N.C. Lin, in Protostars and Planets III, edited by E.H. Levy, J.I. Lunine (1993), pp. 721-748

[2] R.H. Durisen, A.P. Boss, L. Mayer, A.F. Nelson, T. Quinn, W.K.M. Rice, in Protostars and Planets V (2007), pp. 607-622

[3] W.K.M. Rice, G. Lodato, J.E. Pringle, P.J. Armitage, I.A. Bonnell, MNRAS, 372, L9 (2006)

[4] P.G. Gibbons, W.K.M. Rice, G.R. Mamatsashvili, MNRAS, 426, 1444 (2012)

[5] C.F. Gammie, ApJ, 553, 174 (2001)

[6] A.C. Boley, A.C. Mejía, R.H. Durisen, K. Cai, M.K. Pickett, P. D’Alessio, ApJ, 651, 517 (2006)

[7] A.C. Boley, ApJ1, 695, L53 (2009)

[8] G. Bertin, G. Lodato, A\&A, 370, 342 (2001)

[9] W.K.M. Rice, P.J. Armitage, M.R. Bate, I.A. Bonnell, MNRAS, 339, 1025 (2003)

[10] G. Laughlin, P. Bodenheimer, ApJ, 436, 335 (1994)

[11] A.F. Nelson, W. Benz, T.V. Ruzmaikina, ApJ, 529, 357 (2000)

[12] B.M. Johnson, C.F. Gammie, ApJ, 597, 131 (2003)

[13] G. Lodato, W.K.M. Rice, MNRAS, 351, 630 (2004)

[14] A.C. Mejía, R.H. Durisen, M.K. Pickett, K. Cai, ApJ, 619, 1098 (2005)

[15] D. Stamatellos, A.P. Whitworth, A\&A, 480, 879 (2008)

[16] G. Bodo, G. Chagelishvili, G. Murante, A. Tevzadze, P. Rossi, A. Ferrari, A\&A, 437, 9 (2005)

[17] R.V.E. Lovelace, H. Li, S.A. Colgate, A.F. Nelson, ApJ, 513, 805 (1999)

[18] H. Li, S.A. Colgate, B. Wendroff, R. Liska, ApJ, 551, 874 (2001)

[19] M.K. Lin, ApJ, 754, 21 (2012)

[20] H. Meheut, R. Keppens, F. Casse, W. Benz, A\&A, 542, A9 (2012)

[21] H.H. Klahr, P. Bodenheimer, ApJ, 582, 869 (2003)

[22] M.R. Petersen, G.R. Stewart, K. Julien, ApJ, 658, 1252 (2007)

[23] Y. Lithwick, ApJ, 670, 789 (2007)

[24] P. Barge, J. Sommeria, A\&A, 295, L1 (1995)

[25] G.R. Mamatsashvili, W.K.M. Rice, MNRAS, 394, 2153 (2009)

[26] W. Lyra, A. Johansen, A. Zsom, H. Klahr, N. Piskunov, A\&A, 497, 869 (2009)

[27] M.K. Lin, J.C.B. Papaloizou, MNRAS, 415, 1426 (2011)

[28] M.K. Lin, MNRAS, 426, 3211 (2012)

[29] T. Heinemann, J.C.B. Papaloizou, MNRAS, 397, 52 (2009)

[30] S.J. Paardekooper, G. Lesur, J.C.B. Papaloizou, ApJ, 725, 146 (2010)

[31] G.R. Mamatsashvili, G.D. Chagelishvili, MNRAS, 381, 809 (2007)

[32] A. Bracco, P.H. Chavanis, A. Provenzale, E.A. Spiegel, Physics of Fluids 11, 2280 (1999)

[33] P. Godon, M. Livio, ApJ, 523, 350 (1999)

[34] O.M. Umurhan, O. Regev, A\&A, 427, 855 (2004)

[35] B.M. Johnson, C.F. Gammie, ApJ, 635, 149 (2005)

[36] Y. Shen, J.M. Stone, T.A. Gardiner, ApJ, 653, 513 (2006)

[37] G. Bodo, A. Tevzadze, G. Chagelishvili, A. Mignone, P. Rossi, A. Ferrari, A\&A, 475, 51 (2007)

[38] A. Johansen, A.C. Andersen, A. Brandenburg, A\&A, 417, 361 (2004)

[39] S. Fromang, R.P. Nelson, MNRAS, 364, L81 (2005)

[40] W.K.M. Rice, P.J. Armitage, G.R. Mamatsashvili, G. Lodato, C.J. Clarke, MNRAS, 418, 1356 (2011) 